\title{
Variación aloenzimática y parentesco evolutivo en especies de Microlophus del grupo “peruvianus” (Squamata: Tropiduridae)
}

\author{
Allozyme variation and evolutionary relationships in species of the genus \\ Microlophus («peruvianus» group) (Squamata; Tropiduridae)
}

\author{
PEDRO F. VICTORIANO ${ }^{1}$, FERNANDO TORRES-PÉREZ ${ }^{1}$, JUAN CARLOS ORTIZ ${ }^{1}$ \\ LUIS E. PARRA ${ }^{1}$, IRMA NORTHLAND ${ }^{2} \&$ JUANA CAPETILLO $^{2}$ \\ ${ }^{1}$ Departamento de Zoología, Universidad de Concepción, Casilla 160-C, Concepción, Chile;
e-mail: pvictori@udec.cl \\ ${ }^{2}$ Departamento Biomédico, Facultad de Ciencias de la Salud, Universidad de Antofagasta
}

\begin{abstract}
RESUMEN
Se determinó la variación aloenzimática y el grado de parentesco evolutivo en especies del género Microlophus del grupo "peruvianus" provenientes de 11 localidades de Chile y sur de Perú. Además se incluyó a dos grupos externos (M. occipitalis y M. thoracicus, ambos del norte de Perú), con el fin de polarizar los estados de caracteres en un análisis cladístico. Se analizó un total de 22 presuntos loci, de los cuales dieciocho fueron variables. Los resultados obtenidos sugieren la existencia de tres especies en el sur de Perú y norte de Chile. Dos especies habitan en el intermareal; una corresponde a $M$. quadrivittatus que se extiende desde Islay en Perú, hasta Antofagasta por el sur, y la otra, $M$. atacamensis se distribuye desde el sur de Antofagasta hasta Tres Playitas (Huasco). La tercera especie corresponde a M. theresioides, la cual se distribuye en el desierto interior de Chile, abarcando las localidades de Pica, Huayca y Mamiña, y probablemente se extienda hasta el rango costero del desierto chileno. Este ordenamiento taxonómico resultó concordante con los niveles de identidad genética de Rogers, cuyo dendrograma agrupó a las poblaciones en nodos consistentes con las tres especies. El análisis filogenético confirma la existencia de tres clados atribuibles a las especies sugeridas por el análisis fenético. No se encontró evidencia de la monofilia de las dos especies de intermareal, lo que indica que $M$. atacamensis es el taxón hermano del clado $M$. quadrivittatus- $M$. theresioides. De acuerdo a esto, la adaptación al hábitat costero pudo surgir en más de una ocasión en la historia evolutiva de los Microlophus del grupo "peruvianus", o bien corresponde a un atributo plesiomórfico dentro del grupo analizado.
\end{abstract}

Palabras clave: Microlophus, Tropiduridae, variación aloenzimática, relaciones evolutivas, Chile.

\begin{abstract}
Allozyme variation and evolutionary relationship in species of the genus Microlophus of the peruvianus group from 11 localities in Chile and southern Perú were determined. Two external groups (M. occipitalis and M. thoracicus, both from northern Perú), were considered to polarize character states in a cladistics analysis. A total of 22 presumptive loci were analysed, of which 18 were variables. The results obtained suggests the presence of three species of Microlophus in southern Perú and northern Chile. Two species inhabit intertidal areas; one correspond to M. quadrivittatus which extends from Islay in Perú (16 $\left.6^{\circ} 9^{\prime} \mathrm{S}, 7^{\circ} 06^{\prime} \mathrm{W}\right)$ to Antofagasta in Chile (2340' S, 70²6' W). The second species, $M$. atacamensis, is distribuited from the south of Antofagasta to Tres Playitas in Chile ( $\left.28^{\circ} 28^{\prime} \mathrm{S}, 71^{\circ} 14^{\prime} \mathrm{W}\right)$. The third species corresponds to $M$. theresioides, which is distribuited in the chilean desert, in Pica (20³0' S, 69'20' W), Huayca $\left(20^{\circ} 11^{\prime} \mathrm{S}, 69^{\circ} 08^{\prime} \mathrm{W}\right)$, and Mamiña (20 $\left.04^{\circ} \mathrm{S}, 69^{\circ} 13^{\prime} \mathrm{W}\right)$, and probably extends to the coastal range of the Chilean desert at this latitude. The taxonomic arrangement proposed is according to Rogers genetic identity levels, where the respective dendrogram clustered populations in three nodes, one for each species. Phylogenetic analysis confirms the existence of three clades corresponding to species suggested by phenetic analysis. We did not detect evidence of monophily of both coastal species. This indicates that M. atacamensis is the sister taxon of M. quadrivittatus - M. theresioides clade. According to this, the adaptation to an intertidal habitat could have originated more than once in the evolutionary history of Microlophus of the peruvianus group. Another explanation is that this adaptation is plesiomorphic within the group analysed.
\end{abstract}

Key words: Microlophus, Tropiduridae, allozymic variation, evolutionary relationships, Chile.

\section{INTRODUCCIÓN}

Microlophus Duméril y Bibron, 1837, está conformado por especies que se distribuyen en la vertiente occidental de los Andes a lo largo de la franja pacífica del norte de Chile y Perú. Existe evidencia filogenética de su condición monofilética, y de la ocurrencia de dos clados al 
interior del género ("occipitalis" y "peruvianus"), los cuales son claramente diagnosticables por sinapomorfías referidas a escamación, pliegues corporales (Dixon \& Wright 1975) y caracteres osteológicos (Frost 1992). A diferencia del grupo "occipitalis" que se distribuye en forma disjunta en el norte de Perú y en las islas Galápagos, el conjunto "peruvianus" se distribuye exclusivamente en el continente sudamericano (Dixon \& Wright 1975). Tanto la morfología exosomática, como aspectos biológicos han sugerido dentro del grupo "peruvianus" la divergencia entre taxa que viven exclusivamente en la franja de mareas $(M$. quadrivittatus, $M$. atacamensis) y los de interior (M. yanesi, $M$. theresioides, $M$. heterolepis, $M$. peruvianus, $M$. theresiae, $M$. thoracicus, $M$. tigris y M. tarapacensis) (Ortiz \& Serey 1979, Ortiz 1980a).

Dentro del género, la monofilia del clado "peruvianus" es sustentada por evidencia filogenética. Sin embargo, existen varias situaciones taxonómicas confusas al interior de este grupo, donde los límites entre taxa específicos están poco resueltos, dificultando la realización de análisis de parentesco evolutivo entre especies. Por ejemplo, Ortiz (1980b) sugirió a partir de un estudio morfológico para poblaciones de la costa chileno-peruana, la presencia de dos especies: $M$. atacamensis, distribuida desde Tres Playitas en Chile ( $28^{\circ} 28^{\prime} \mathrm{S}, 71^{\circ} 14^{\prime} \mathrm{O}$ ), hasta las cercanías del río Loa $\left(22^{\circ} 16^{\prime} \mathrm{S}, 70^{\circ} 05^{\prime} \mathrm{O}\right)$, y $M$. quadrivittatus, presente desde el río Loa hasta Islay en Perú. Sin embargo, estudios cariotípicos realizados por Northland et al. (1987) no mostraron diferencias entre ambos taxa, sugeriendo que éstos corresponden a una única especie a lo largo del litoral chileno-peruano.

Por otra parte, existe confusión respecto a la delimitación de las especies que habitan en el interior. Por ejemplo, aún no ha sido aclarada la situación taxonómica de las poblaciones que existen en las cerranías costeras, pero que no habitan el intermareal. Para el desierto interior, se ha sugerido la existencia de al menos tres especies: una remitida a la quebrada de Mamiña, con cariotipos que difieren de las poblaciones aledañas (M. maminensis) (Northland et al. 1987), una segunda especie ( $M$. theresioides), con localidad tipo en el oasis de Pica, y una tercera especie, $M$. tarapacensis, descrita para el desierto de Tarapacá (Donoso-Barros 1966).

La hipótesis de la existencia de dos grupos monofiléticos, uno conformado por especies de intermareal y otro por las del desierto interior, no ha sido puesta a prueba mediante un análisis cladístico formal. Una herramienta útil para responder interrogantes sistemático-evolutivas en casos de difícil resolución a partir de estudios morfológicos, es el uso de caracteres aloenzimáticos. Estos pueden ser analizados en un contexto filogenético, de manera que las hipótesis taxonómicas reflejen los grados de parentesco entre taxa (e.g., Martins 1995). La técnica electroforética genera información acerca de los tipos de alelos y sus frecuencias, permitiendo estimar distancias genéticas entre taxa (Nei 1978). A su vez, las variantes alélicas pueden ser consideradas como estados de caracteres en un análisis cladístico (Buth 1984). El cladismo se basa en la existencia de grupos que se originaron a partir de un ancestro común (monofiléticos), y en cuyos taxa derivados han surgido caracteres nuevos o apomórficos (Wiley 1981). Para hipotetizar acerca de los estados de caracteres en derivados o ancestrales, es necesario incluir uno o más grupos externos en este análisis, generalmente un taxón perteneciente a un grupo hermano.

En este trabajo se informan los resultados de un análisis de varición aloenzimática en Microlophus del grupo "peruvianus" del sur de Perú y norte de Chile, con el fin de definir el estatus taxonómico de sus poblaciones. Además se realizó un análisis filogenético considerando las variantes alélicas como estados de caracteres, con el fin de proponer hipótesis sobre la divergencia evolutiva y las relaciones de parentesco entre los grupos estudiados.

\section{MATERIALES Y MÉTODOS}

Se analizaron ejemplares provenientes de 11 localidades, dos de Perú y nueve de Chile (Fig.1). Las localidades consideradas y los números de ejemplares analizados son los siguientes: Ilo (roqueríos intermareales en el sur de Perú, 17³8' $\mathrm{S}, 71^{\circ} 20^{\prime} \mathrm{O}, \mathrm{n}=5$ ); Caleta Mecca, (intermareal de la costa sur de Perú, $\left.17^{\circ} 55^{\prime} \mathrm{S}, 70^{\circ} 51^{\prime} \mathrm{O}, \mathrm{n}=11\right)$; Arica (Playa Corazones, $18^{\circ} 28^{\prime}$ S, 70¹9' O, n = $16)$; Iquique ( $M$. quadrivittatus, intermareal rocoso, 20 $0^{\circ} 13^{\prime} \mathrm{S}, 70^{\circ} 10^{\prime} \mathrm{O}, \mathrm{n}=41$ ); Mamiña (affinis theresioides, $20^{\circ} 04^{\prime} \mathrm{S}, 6^{\circ} 13^{\prime} \mathrm{O}, \mathrm{n}=6$ ); Huayca (affinis theresioides, $20^{\circ} 11^{\prime} \mathrm{S}, 6^{\circ} 08^{\prime} \mathrm{O}, \mathrm{n}=11$ ); Pica (M. theresioides, 20³0' S, 69'20' O, $\mathrm{n}=$ 11); Río Loa (intermareal en la desembocadura, $21^{\circ} 20^{\prime} \mathrm{S}, 70^{\circ} 40^{\prime} \mathrm{O}, \mathrm{n}=10$ ); Antofagasta 1 (laderas de cerros costeros $23^{\circ} 36^{\prime} \mathrm{S}, 7^{\circ} 22^{\prime} \mathrm{O}, \mathrm{n}=20$ ); Antofagasta 2 (intermareal rocoso, $23^{\circ} 40^{\prime} \mathrm{S}$, $\left.70^{\circ} 26^{\prime} \mathrm{O}, \mathrm{n}=17\right)$ y Caldera $(M$. atacamensis, $27^{\circ} 40^{\prime} \mathrm{S}, 70^{\circ} 49^{\prime} \mathrm{O}, \mathrm{n}=24$ ).

Los ejemplares fueron trasladados vivos al laboratorio, o disecados directamente en el campo, extrayéndoles tejido muscular y hepático. Los tejidos fueron congelados en nitrógeno líquido, y 
posteriormente mantenidos en laboratorio a -80 ${ }^{\circ} \mathrm{C}$, hasta la realización de los análisis electroforéticos. Las muestras fueron homogeneizadas según Murphy et al. (1996). Las electroforesis se realizaron según Selander et al. (1971) y Murphy et al. (1996), usando como soporte almidón al $12 \%$. Los tipos de tampones utilizados para cada sistema enzimático se basaron en Victoriano et al (1995). El tiempo de corrida electroforética fluctuó entre 5 y $7 \mathrm{~h}$, dependiendo del sistema a revelar. Se obtuvo resultados interpretables para un total de 22 presuntos loci. Se usaron individuos de referencia y comparaciones de "lado a lado" para identificar los electromorfos, los cuales fueron identificados con el sistema alfabético (Richardson et al. 1986). Los genotipos fueron determinados según Harris \& Hopkinson (1976). Los datos de frecuencias alélicas, niveles de variabilidad (heterocigosis

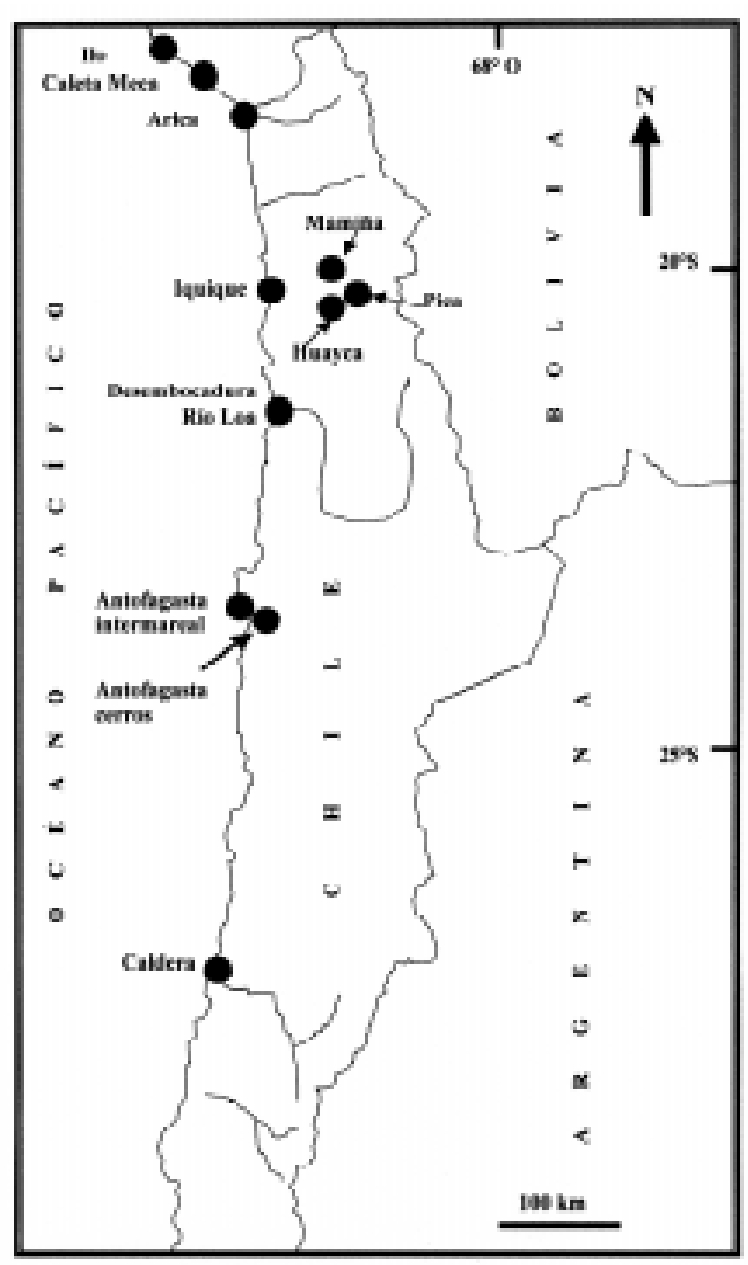

Fig 1: Ubicación de los sitios de muestreo de Microlophus.

Location of sample sites of Microlophus. promedio, número de alelos por locus y porcentaje de loci polimórficos), fueron calculados mediante el programa POPGENE-1.21 (Yeh et al. 1997). Los valores de identidad genética fueron estimados mediante el índice de Rogers (1972) usando el programa TFPGA 1.3 (Miller 2000). Se optó por este índice por ser un estimador métrico que calcula el promedio de las distancias entre dos poblaciones para cada locus en un espacio $\mathrm{k}$ dimensional (con $\mathrm{k}$ alelos). A partir de este índice, la posición de cada población está determinada por un punto, cuyas coordenadas son las frecuencias de los $\mathrm{k}$ alelos de cada locus. Este índice, a diferencia de otros, siempre entrega distancias genéticas iguales para comparaciones que presentan iguales diferencias entre frecuencias génicas (Rogers 1972). A partir de los valores de identidad genética se construyó un dendrograma según el método UPGMA, con el fin de obtener asociaciones fenéticas entre grupos. En este caso, se realizó un análisis de bootstrap de 1.000 permutaciones, con el fin de estimar la probabilidad de ocurrencia de cada nodo. Los estimadores de variabilidad (Heterocigosis, promedio de alelos por locus y porcentaje de polimorfismo), fueron calculados sólo para las poblaciones representadas por más de 10 ejemplares. Los loci que presentaron alelos fijados no compartidos entre dos o más grupos fueron considerados como diagnóstico (criterio de $95 \%$ de frecuencia para el alelo más común).

En el análisis cladístico se utilizaron dos grupos externos, con el fin de polarizar los estados de caracteres en plesiomórficos (primitivos) o apomórficos (derivados). El primer grupo externo correspondió a $M$. occipitalis de la localidad de Jequetepeque (Perú, 07²19' S, 79³4' O, tres ejemplares) por pertenecer al grupo hermano del metataxón "peruvianus". Se eligió como segundo grupo externo a $M$. thoracicus, de Puerto Morín, Perú ( $8^{\circ} 24^{\prime}$ S, 78 54' O, cinco ejemplares), por ser la especie que más difiere fenéticamente al interior del grupo "peruvianus" (Mertens 1956, Dixon \& Wright 1975). En la codificación se consideró a cada alelo como carácter, asignando el estado plesiomórfico al presente en el grupo externo. Esta modalidad de codificación fue propuesta por Mickevich \& Mitter (1981), el cual es denominado "modelo del alelo independiente". El razonamiento de esta opción es que en el proceso evolutivo los alelos pueden adquirirse o perderse como eventos independientes. En los casos de loci polimórficos, se siguió el método de asignación de rangos para diferentes frecuencias sugerido por Wiens (1993). Este consiste en que los alélos de loci polimórficos son asignados a uno de cuatro estados discretos (0-3) basados en 
TABLA 1

Frecuencias alélicas para los loci variables en poblaciones de Microlophus. M occip.: M. occipitalis de Jequetepeque (Perú). M. thorac.: M. thoracicus de Puerto Morín (Perú). Bajo cada localidad se indica el número de individuos analizados

Allelic frequencies for variable loci in populations of Microlophus. M occip.: M. occipitalis from Jequetepeque

(Perú). M. thorac.: M. thoracicus from Puerto Morín (Perú). Below sample sites the number of individuals is indicated

\begin{tabular}{|c|c|c|c|c|c|c|c|c|c|c|c|c|c|}
\hline \multirow[t]{3}{*}{ Locus/alelo } & \multirow[t]{2}{*}{ M. occip } & \multirow[t]{2}{*}{ M.thorac. } & \multirow[t]{2}{*}{ Ilo } & \multirow[t]{2}{*}{ C. Mecca } & \multirow[t]{2}{*}{ Arica } & \multirow[t]{2}{*}{ Iquique } & \multirow[t]{2}{*}{ Río Loa } & \multicolumn{2}{|c|}{ Antofagasta } & \multirow[t]{2}{*}{ Caldera } & \multirow[t]{2}{*}{ Pica } & \multirow[t]{2}{*}{ Huayca } & \multirow[t]{2}{*}{ Mamiña } \\
\hline & & & & & & & & 1 & 2 & & & & \\
\hline & 3 & 5 & 5 & 11 & 16 & 41 & 10 & 20 & 17 & 24 & 11 & 11 & 6 \\
\hline \multicolumn{14}{|l|}{ LDH-1 } \\
\hline A & 1,000 & 1,000 & 1,000 & 1,000 & 1,000 & 1,000 & 1,000 & 1,000 & 1,000 & 1,000 & 0,825 & 1,000 & 1,000 \\
\hline B & & & & & & & & & & & 0,175 & & \\
\hline \multicolumn{14}{|l|}{ LDH-2 } \\
\hline A & & 1,000 & 1,000 & 1,000 & 1,000 & 0,980 & 1,000 & 1,000 & 1,000 & 1,000 & 1,000 & 1,000 & 1,000 \\
\hline B & 1,000 & & & & & & & & & & & & \\
\hline $\mathrm{C}$ & & & & & & 0,020 & & & & & & & \\
\hline \multicolumn{14}{|l|}{ EST-1 } \\
\hline A & & & 1,000 & 1,000 & 1,000 & 0,969 & 1,000 & 0,950 & 1,000 & & 0,550 & 0,364 & 0,438 \\
\hline B & & & & & & 0,020 & & 0,050 & & 1,000 & & 0,091 & 0,063 \\
\hline $\mathrm{C}$ & 1,000 & & & & & & & & & & 0,450 & 0,409 & 0,500 \\
\hline D & & & & & & & & & & & & 0,091 & \\
\hline E & & 1,000 & & & & & & & & & & 0,046 & \\
\hline $\mathrm{F}$ & & & & & & 0,010 & & & & & & & \\
\hline \multicolumn{14}{|l|}{ EST-2 } \\
\hline A & & 1,000 & 1,000 & 1,000 & 1,000 & 1,000 & 1,000 & 1,000 & 1,000 & 1,000 & 1,000 & 1,000 & 1,000 \\
\hline B & 1,000 & & & & & & & & & & & & \\
\hline \multicolumn{14}{|l|}{ AAT-1 } \\
\hline A & & 1,000 & 0,500 & & 1,000 & 1,000 & 0,950 & 1,000 & 1,000 & 1,000 & 0,921 & 1,000 & 1,000 \\
\hline B & & & & & & & & & & & 0,026 & & \\
\hline $\mathrm{C}$ & 1,000 & & 0,500 & 1,000 & & & 0,050 & & & & 0,026 & & \\
\hline D & & & & & & & & & & & 0,026 & & \\
\hline \multicolumn{14}{|l|}{ AAT-2 } \\
\hline A & & 1,000 & 1,000 & 1,000 & 0,875 & 1,000 & 1,000 & 0,700 & 0,158 & & 0,790 & 0,364 & 0,556 \\
\hline B & & & & & & & & 0,200 & 0,158 & 0,786 & & & \\
\hline $\mathrm{C}$ & & & & & & & & 0,100 & 0,632 & 0,214 & & & \\
\hline D & & & & & & & & & 0,053 & & 0,211 & 0,636 & 0,444 \\
\hline E & & & & & 0,125 & & & & & & & & \\
\hline $\mathrm{F}$ & 1,000 & & & & & & & & & & & & \\
\hline \multicolumn{14}{|l|}{ IDH-1 } \\
\hline A & & & 0,800 & 0,818 & 1,000 & 0,941 & 0,950 & 0,947 & 0,912 & 0,938 & 1,000 & 0,900 & 1,000 \\
\hline B & & & & 0,091 & & 0,048 & & & & 0,021 & & 0,050 & \\
\hline $\mathrm{C}$ & & & 0,100 & 0,091 & & 0,012 & 0,050 & 0,053 & 0,088 & 0,042 & & 0,050 & \\
\hline D & & 1,000 & & & & & & & & & & & \\
\hline E & 1,000 & & & & & & & & & & & & \\
\hline $\mathrm{F}$ & & & 0,100 & & & & & & & & & & \\
\hline \multicolumn{14}{|l|}{ IDH-2 } \\
\hline A & 1,000 & & 1,000 & 1,000 & 1,000 & 1,000 & 1,000 & 1,000 & 1,000 & 1,000 & 1,000 & 1,000 & 1,000 \\
\hline B & & 1,000 & & & & & & & & & & & \\
\hline
\end{tabular}


TABLA 1 (continuación)

\begin{tabular}{|c|c|c|c|c|c|c|c|c|c|c|c|c|c|}
\hline \multirow[t]{3}{*}{ Locus/alelo } & \multirow[t]{2}{*}{ M. осcip } & \multirow[t]{2}{*}{ M.thorac. } & \multirow[t]{2}{*}{ Ilo } & \multirow[t]{2}{*}{ C. Mecca } & \multirow[t]{2}{*}{ Arica } & \multirow[t]{2}{*}{ Iquique } & \multirow[t]{2}{*}{ Río Loa } & \multicolumn{2}{|c|}{ Antofagasta } & \multirow[t]{2}{*}{ Caldera } & \multirow[t]{2}{*}{ Pica } & \multirow[t]{2}{*}{ Huayca } & \multirow[t]{2}{*}{ Mamiña } \\
\hline & & & & & & & & 1 & 2 & & & & \\
\hline & 3 & 5 & 5 & 11 & 16 & 41 & 10 & 20 & 17 & 24 & 11 & 11 & 6 \\
\hline \multicolumn{14}{|l|}{ GPD-1 } \\
\hline A & & 1,000 & 1,000 & 1,000 & 1,000 & 1,000 & 1,000 & 1,000 & 1,000 & 1,000 & 1,000 & 1,000 & 1,000 \\
\hline B & 1,000 & & & & & & & & & & & & \\
\hline \multicolumn{14}{|l|}{ MDHP-1 } \\
\hline A & & & 0,600 & 0,917 & 0,875 & 0,918 & 1,000 & 0,211 & 0,523 & & 0,118 & 0,700 & 0,438 \\
\hline B & & & & & 0,125 & 0,061 & & 0,500 & 0,318 & 1,000 & 0,647 & 0,100 & 0,375 \\
\hline $\mathrm{C}$ & & & & & & & & & & & 0,118 & & \\
\hline $\mathrm{D}$ & & 1,000 & & & & & & 0,237 & 0,046 & & & & 0,063 \\
\hline $\mathrm{E}$ & & & & & & 0,020 & & 0,053 & 0,114 & & & 0,100 & \\
\hline $\mathrm{F}$ & & & 0,400 & 0,083 & & & & & & & 0,118 & 0,100 & 0,125 \\
\hline G & 1,000 & & & & & & & & & & & & \\
\hline \multicolumn{14}{|l|}{ PGM-1 } \\
\hline A & & 0,900 & 1,000 & 1,000 & 1,000 & 1,000 & 1,000 & 0,950 & 1,000 & 1,000 & 1,000 & 1,000 & 1,000 \\
\hline B & & & & & & & & 0,050 & & & & & \\
\hline $\mathrm{C}$ & 1,000 & & & & & & & & & & & & \\
\hline $\mathrm{D}$ & & 0,100 & & & & & & & & & & & \\
\hline \multicolumn{14}{|l|}{ PGM-2 } \\
\hline A & & 1,000 & 1,000 & 1,000 & 1,000 & 1,000 & 1,000 & 1,000 & 1,000 & 1,000 & 1,000 & 1,000 & 1,000 \\
\hline B & 1,000 & & & & & & & & & & & & \\
\hline \multicolumn{14}{|l|}{ LAP-1 } \\
\hline A & & 1,000 & 1,000 & 1,000 & 1,000 & 1,000 & 1,000 & 1,000 & 1,000 & 1,000 & 1,000 & 1,000 & 1,000 \\
\hline B & 1,000 & & & & & & & & & & & & \\
\hline \multicolumn{14}{|l|}{ OPDH-1 } \\
\hline A & 1,000 & 1,000 & 1,000 & 1,000 & 1,000 & 1,000 & 1,000 & 0,950 & 1,000 & 1,000 & 1,000 & 1,000 & 1,000 \\
\hline B & & & & & & & & 0,050 & & & & & \\
\hline \multicolumn{14}{|l|}{ ESTD-1 } \\
\hline A & & & & & & & & & & 1,000 & & & \\
\hline B & & & 1,000 & 1,000 & 1,000 & 1,000 & 1,000 & 1,000 & 1,000 & & 0,688 & 1,000 & 1,000 \\
\hline $\mathrm{C}$ & & & & & & & & & & & 0,313 & & \\
\hline $\mathrm{D}$ & 1,000 & & & & & & & & & & & & \\
\hline $\mathrm{E}$ & & 1,000 & & & & & & & & & & & \\
\hline ESTD-2 & & & & & & & & & & & & & \\
\hline A & & & 1,000 & 1,000 & 1,000 & 1,000 & 1,000 & 1,000 & 1,000 & 1,000 & 1,000 & 1,000 & 1,000 \\
\hline B & & 1,000 & & & & & & & & & & & \\
\hline $\mathrm{C}$ & 1,000 & & & & & & & & & & & & \\
\hline PGI-1 & & & & & & & & & & & & & \\
\hline A & 1,000 & & 1,000 & 1,000 & 1,000 & 1,000 & 0,500 & 1,000 & 0,900 & & 1,000 & 1,000 & 1,000 \\
\hline B & & & & & & & 0,500 & & 0,100 & 1,000 & & & \\
\hline $\mathrm{C}$ & & 1,000 & & & & & & & & & & & \\
\hline GLY-1 & & & & & & & & & & & & & \\
\hline A & 1,000 & 1,000 & 1,000 & 1,000 & 1,000 & 0,955 & 1,000 & 1,000 & 1,000 & 1,000 & 1,000 & 1,000 & 1,000 \\
\hline B & & & & & & 0,046 & & & & & & & \\
\hline
\end{tabular}


los rangos de frecuencias alélicas. Para esto se hizo el supuesto de que un carácter derivado sigue una vía a través de estados de polimorfismo antes de llegar a ser fijados, y que los polimorfismos pueden ser mantenidos a través de los eventos de especiación (Wiens 2000, Wiens \& Servedio 2000). En este caso, el ordenamiento de las series de transformación sigue un criterio de parsimonia, ubicando las categorías de manera que la magnitud de evolución alélica sea la menor (Buth 1984). Los datos fueron analizados usando el programa NONA 2.0 (Goloboff 1993) ejecutado por medio de WINCLADA 0.9.99.50.mexu (BETA) (Nixon 2002), para lo cual se consideraron sólo aquellos caracteres informativos, es decir, que presentaban variantes alélicas al menos en uno de los grupos analizados. El análisis consideró a todos los caracteres como ordenados. La matriz (Anexos 1 y 2) fue analizada mediante 50 replicas de secuencia de adición al azar, con permutación de ramas de tipo "tree bisection reconnection” (TBR) (Swofford \& Olsen 1990). El apoyo de los grupos resultantes se evaluó con "parsimony jacknifing" (Farris et al. 1997) con 100 iteraciones, con el fin de estimar la probabilidad de obtener cada uno de los ordenamientos filogenéticos del análisis.

\section{RESULTADOS}

De los 22 loci registrados, 18 mostraron variantes alélicas al menos para una de las localidades analizadas (LDH-1, LDH-2, EST-1, EST-2, AAT1, AAT -2, IDH-1, IDH-2, GPD-1, MDHP-1, PGM-1, PGM-2, LAP-1, OPDH-1, ESTD-1, ESTD-2, PGI-1 y GLY-1) (Tabla 1). Los loci con un mayor número de alelos fueron la EST-1 (seis alelos ), la AAT-2 (seis alelos ), la IDH-1 (seis alelos) y la MDHP-1 (siete alelos). Del total de loci variables, 11 se presentaron como diagnósticos para la especie M. occipitalis (LDH-2, EST2, AAT-2, IDH-1, GPD-1, MDHP-1, PGM-1, PGM-2, LAP-1, ESTD-1, ESTD-2), mientras que $M$. thoracicus, el segundo grupo externo del análisis filogenético, mostró seis loci con esta condición (EST-1, IDH-1, IDH-2, ESTD-1, ESTD-2, y la PGI-1). Dentro del grupo de intermareal, la localidad de Caldera (M. atacamensis) mostró tres loci con un alelo fijado ausente en las demás localidades de intermareal (EST-1, ESTD-1 y PGI-1) (caracteres diagnóstico según criterio de $95 \%$ ). Otros dos loci mostraron notables diferencias en las frecuencias alélicas entre Caldera y las demás localidades de intermareal aunque sin ser caracteres diagnóstico (AAT-2 y MDHP-1).

Las poblaciones peruanas de Ilo y Caleta Mecca comparten un alelo frecuente del locus AAT-1 con M. occipitalis (alelo C), el cual en las demás localidades está ausente o en baja frecuencia. Respecto a las localidades de interior, las poblaciones de Pica, Mamiña y Huayca mostraron un alelo frecuente en los loci EST-1 y AAT-2 inexistente en las demás poblaciones (alelo D).

Las poblaciones de interior tienden a ser más variables genéticamente que las de intermareal (Tabla 2). La localidad de Pica mostró valores comparativamente altos para los tres indicadores, y dos de las localidades de interior (Antofagasta 1 y Pica) mostraron el polimorfismo más alto $(22,7 \%)$. Entre las localidades de intermareal, tres localidades ubicadas en los extremos del rango geográfico estudiado (Caleta Mecca y Arica en el extremo norte, y Caldera en el sur) mostraron bajos niveles de variabilidad genética.

De acuerdo a la matriz de distancias genéticas (Tabla 3), los menores niveles de distancia se encuentran tanto en las comparaciones entre las localidades costeras del norte de Chile (Arica, Iquique, y Río Loa), como entre las de interior

TABLA 2

Variabilidad genética para los Microlophus analizados. Sólo se informan las localidades representadas por diez o más ejemplares

Genetic variability levels for Microlophus analyzed. Only localities represented by more than ten individuals are shown

\begin{tabular}{|c|c|c|c|c|c|c|c|c|c|}
\hline \multirow[t]{3}{*}{ Indicador de variabilidad } & \multicolumn{9}{|c|}{ Loc a lidad } \\
\hline & \multirow[t]{2}{*}{ Caleta Meca } & \multirow[t]{2}{*}{ Arica } & \multirow[t]{2}{*}{ Iquique } & \multirow[t]{2}{*}{ Río Loa } & \multicolumn{2}{|c|}{ Antofagasta } & \multirow[t]{2}{*}{ Caldera } & \multirow[t]{2}{*}{ Pica } & \multirow[t]{2}{*}{ Huayca } \\
\hline & & & & & 1 & 2 & & & \\
\hline Promedio alelos/ locus & 1,111 & 1,074 & 1,296 & 1,111 & 1,333 & 1,296 & 1,111 & 1,370 & 1,370 \\
\hline Error estándar & 0,424 & 0,267 & 0,669 & 0,320 & 0,734 & 0,823 & 0,424 & 0,839 & 1,006 \\
\hline Promedio heterocigosis & 0,007 & 0,005 & 0,008 & 0,044 & 0,017 & 0,020 & 0,005 & 0,018 & 0,024 \\
\hline Error estándar & 0,035 & 0,024 & 0,024 & 0,193 & 0,046 & 0,053 & 0,018 & 0,048 & 0,106 \\
\hline Polimorfismo (\%) & 7,410 & 7,410 & 18,520 & 11,110 & 22,220 & 14,810 & 7,410 & 22,220 & 14,810 \\
\hline
\end{tabular}


Pica, Huayca y Mamiña. Los niveles más altos de distancia genética correspondieron tanto a las comparaciones entre $M$. occipitalis y las restantes localidades (rangos para distancia $=0,440-0,554$ ), como a las comparaciones entre $M$. thoracicus y el resto. Sin embargo, esta última presentó distancias menores que las comparaciones de $M$. occipitalis (rangos de distancia $=0,260-0,296$ ). Todas las comparaciones que involucraron sólo localidades del grupo interno del análisis cladista, mostraron distancias genéticas menores que las anteriores (rangos de distancia $=0,011-0,221$ ). De estas últimas asociaciones, los valores más altos de distancia genética fueron para las comparaciones entre Caldera y las demás localidades.

El dendrograma de la Fig. 2 muestra, en un orden decreciente de divergencia genética, las siguientes asociaciones: $M$. occipitalis de Jequetepeque mostró las mayores diferencias en composición y frecuencias alélicas (distancia promedio de Rogers $=0,49$ ), mientras que $M$. thoracicus de Puerto Morín se asocia a las restantes localidades con un promedio de distancia de 0,28 . Los demás grupos se ordenaron en dos conjuntos: una primera rama corresponde a la localidad de Caldera, asociada al resto con un promedio de distancia genética de 0,18. Las localidades restantes formaron, por una parte, un grupo compuesto por las tres localidades de interior (Pica, Huayca y Mamiña), y por otro, las localidades costeras del norte de Chile y sur de Perú (Arica, Iquique, Río Loa, Antofagasta 1, Antofagasta 2, Ilo y Caleta Meca). La localidad de Antofagasta 1 (cerros costeros) mostró más semejanzas con las localidades de intermareal que con las de interior.

El análisis cladístico encontró cuatro árboles de máxima parsimonia, con una longitud de 117,

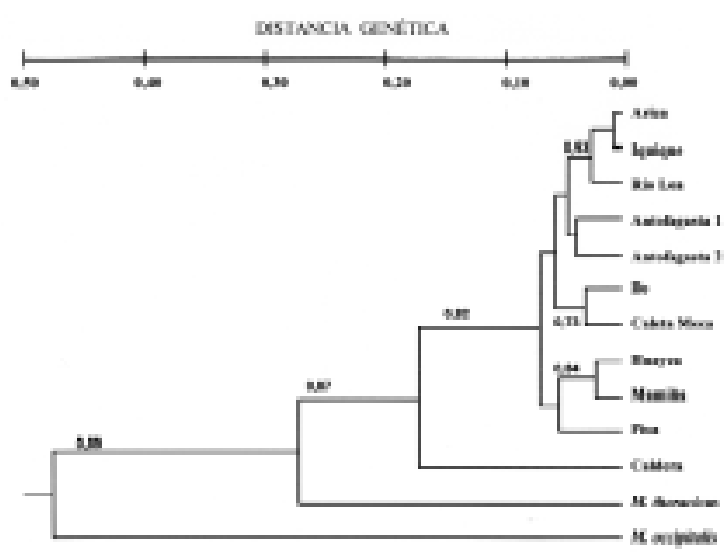

Fig. 2: Fenograma (UPGMA) de distancias genéticas de Rogers (1972), para Microlophus provenientes de las 13 localidades analizadas. Los números sobre los nodos indican valores de bootstrap.

UPGMA phenogram based on Rogers (1972) distances between Microlophus from 13 localities analyzed. Numbers on nodes correspond to bootstrap values.

un índice de consistencia de 0,76, un índice de retención de 0,58 y un ajuste rescalado de 44,08 $\%$. La Fig. 3 muestra el árbol de consenso estricto (longitud 122, índice de consistencia 0,72 e índice de retención 0,51). Las poblaciones analizadas forman un grupo monofilético (53\% de apoyo Jacknifing) sustentado por tres sinapomorfías: 14(3) alelo A del locus IDH-1 con una frecuencia entre 0,76 y 1,$00 ; 20(1)$ alelo B del locus MDHP1 con frecuencia entre 0,26 y 0,50 ; y 33(2) alelo A del locus ESTD-2 (Anexo 1, Anexo 2). El análisis del grupo interno muestra una dicotomia basal que da origen a dos grupos bien apoyados

TABLA 3

Matriz de distancia genética de Rogers (1972) para los Microlophus analizados

Rogers' (1972) genetic distance for populations of Microlophus analysed

\begin{tabular}{|c|c|c|c|c|c|c|c|c|c|c|c|c|}
\hline & \multirow{2}{*}{\multicolumn{2}{|c|}{ M. occip. M. thorac. }} & \multirow[t]{2}{*}{ Ilo } & \multirow[t]{2}{*}{ Caleta } & \multirow[t]{2}{*}{ Arica } & \multirow[t]{2}{*}{ Iquique } & \multirow[t]{2}{*}{ Río Loa } & \multicolumn{2}{|c|}{ Antofagasta } & \multirow[t]{2}{*}{ Caldera } & \multirow[t]{2}{*}{ Pica } & \multirow[t]{2}{*}{ Huayca } \\
\hline & & & & & & & & 1 & 2 & & & \\
\hline M. occip. & - & & & & & & & & & & & \\
\hline M. thorac. & 0,5538 & - & & & & & & & & & & \\
\hline Ilo & 0,4549 & 0,2734 & - & & & & & & & & & \\
\hline Caleta Meca & 0,4400 & 0,2955 & 0,0338 & - & & & & & & & & \\
\hline Arica & 0,4773 & 0,2655 & 0,0427 & 0,0516 & - & & & & & & & \\
\hline Iquique & 0,4781 & 0,2606 & 0,0390 & 0,0455 & 0,0105 & - & & & & & & \\
\hline Río Loa & 0,4973 & 0,2590 & 0,0549 & 0,0611 & 0,0315 & 0,0264 & - & & & & & \\
\hline Antofagasta 1 & 0,4695 & 0,2598 & 0,0593 & 0,0803 & 0,0366 & 0,0395 & 0,0611 & - & & & & \\
\hline Antofagasta 2 & 0,4720 & 0,2805 & 0,0680 & 0,0859 & 0,0436 & 0,0485 & 0,0614 & 0,0414 & - & & & \\
\hline Caldera & 0,5141 & 0,2956 & 0,2004 & 0,2214 & 0,1770 & 0,1801 & 0,1659 & 0,1533 & 0,1518 & - & & \\
\hline Pica & 0,4565 & 0,2651 & 0,0882 & 0,1106 & 0,0684 & 0,0735 & 0,0933 & 0,0592 & 0,0829 & 0,1538 & — & \\
\hline Huayca & 0,4519 & 0,2720 & 0,0754 & 0,0902 & 0,0509 & 0,0519 & 0,0750 & 0,0621 & 0,0569 & 0,1620 & 0,0669 & - \\
\hline Mamiña & 0,4504 & 0,2658 & 0,0742 & 0,0952 & 0,0482 & 0,0538 & 0,0765 & 0,0469 & 0,0544 & 0,1548 & 0,0443 & 0,0249 \\
\hline
\end{tabular}


por el análisis de Jacknifing (78 \%). Esta dicotomia basal separa los individuos pertenecientes a la localidad de Caldera con cinco autapomorfias y un grupo monofilético constituido por las poblaciones localizadas al norte de Caldera, incluidas las de interior. Estas últimas comparten cuatro sinapomorfías: 2(2) alelo A del locus EST-1 con una frecuencia entre 0,51 y $0.75 ; 9(2)$ alelo A del locus AAT- 2 con frecuencia entre 0,51 y 0,75 ; 19(1) alelo A del locus MDHP-1 con una frecuencia entre 0,26 y 0,$50 ;$ y $29(3)$ alelo B del locus ESTD-1 con frecuencia entre 0,76 y 1,00. En el árbol de consenso estricto, las poblaciones al norte de Caldera (Fig. 3), se separan en dos grupos monofiléticos; éstos son: (1) Arica+Iquique+Loa+Ilo+Mecca, cuya monofilia está sustentada por la sinapomorfía 19(3) alelo A del locus MDHP-1 con una frecuencia entre 0,76 y 1,00; y (2) Huayca+Mamiña (apoyo Jacknifing del $59 \%)$, los que comparten la sinapomorfía 2(1) alelo A del locus EST-1 frecuencia entre 0,26 y 0,50 ; y 12(1) alelo D del locus AAT-2 con frecuencia entre 0,26 y 0,50 . Antofagasta 1 (cerros costeros), Antofagasta 2 (intermareal) y Pica, junto a los dos grupos monofiléticos señalados anteriormente, no aparecen resueltos formando una politomía. De los cuatro árboles de máxima parsimonia, el árbol 1 (Fig. 4) resolvió todos los grupos terminales y asociando grupos afines geográficamente. Este permite distinguir dentro del grupo interno a tres clados: en primer lugar se separa la población de Caldera de las litorales del norte más las de interior. A diferencia del árbol de consenso, las tres localidades de interior (Pica, Huayca y Mamiña) conforman un grupo monofilético con cada una de sus poblaciones resueltas. Este grupo es sustentado por una sinapomorfía (EST-1, alelo C, frecuencia 0,490,25). Al igual que en el análisis fenético, la población de Antofagasta 1 (cerros costeros) es asociada al grupo de intermareal del norte.

\section{DISCUSIÓN}

Los resultados obtenidos sugieren que los Microlophus estudiados corresponden a tres especies distintas. Tanto el análisis fenético como el cladístico mostró una diferenciación clara de tres grupos, correspondientes, por una parte, a la

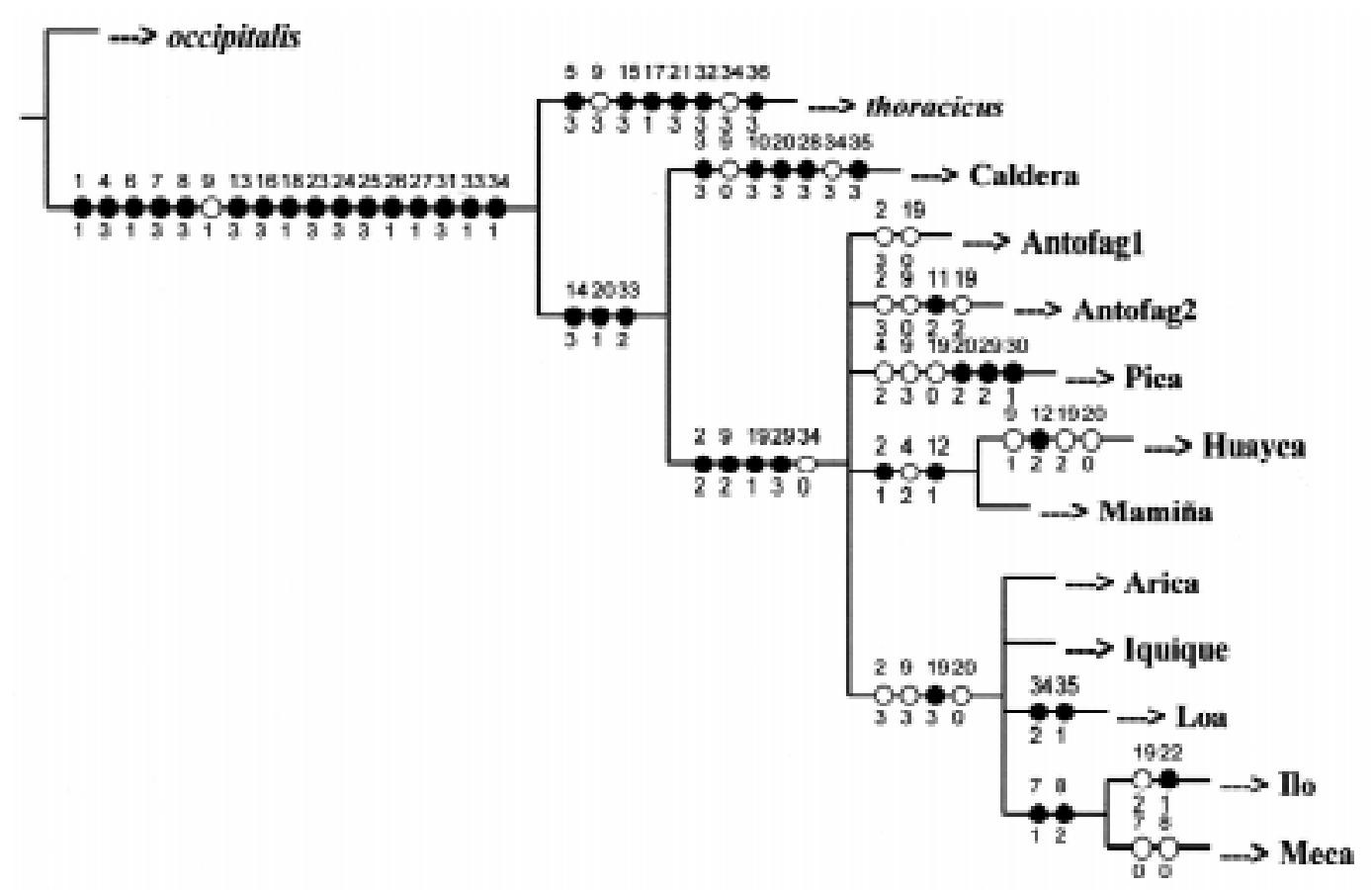

Fig. 3: Arbol de consenso estricto a partir de cuatro igualmente parsimoniosos resultantes de una búsqueda exhaustiva, para 13 poblaciones de Microlophus, basado en 36 caracteres aloenzimáticos. Los números en los nodos corresponden a las apomorfías detalladas en el Anexo 1. Sobre el nodo: número del carácter. Bajo el nodo: estado del carácter. Círculos negros: apomorfías. Círculos blancos: homoplasias.

Strict consensus of the four equally parsimonious trees that resulted from an exhaustive search, for 13 populations of Microlophus, based in 36 allozyme characters. Numbers in nodes correspond to apomorphies detailed in the Appendix 1. Above the node: character number. Below the node: character state. Dark circles: apomorphies. Open circles: homoplasies. 


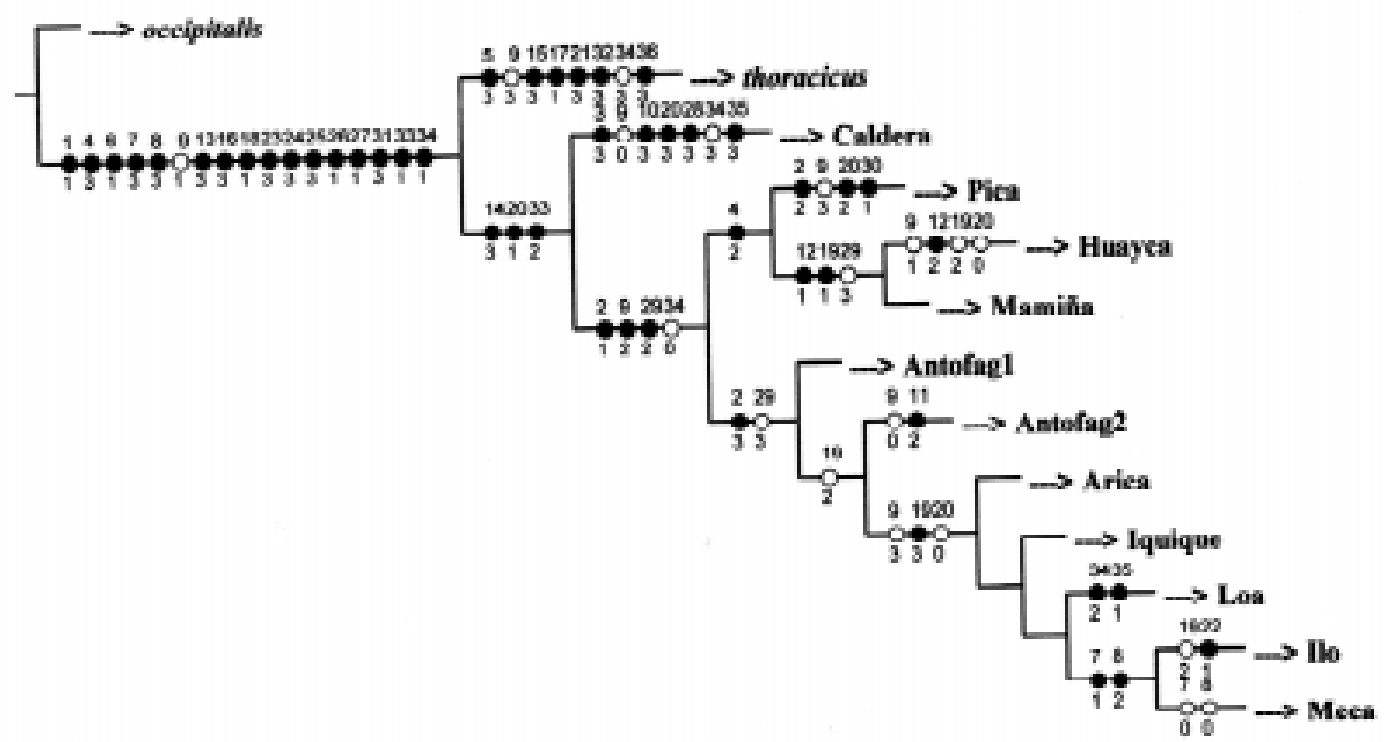

Fig. 4: Uno de los cuatro árboles igualmente parsimoniosos resultantes de una búsqueda exhaustiva, para 13 poblaciones de Microlophus, basado en 36 caracteres aloenzimáticos. Los números sobre los nodos corresponden a las apomorfías detalladas en el Anexo 1. Sobre el nodo: número del carácter. Bajo el nodo: estado del carácter. Círculos negros: apomorfías. Círculos blancos: homoplasias.

Single tree of four equally parsimonious that resulted from an exhaustive search for 13 populations of Microlophus, based in 36 allozyme characters. Numbers in nodes correspond to apomorphies detailed in Appendix 1. Above the node: character number. Below the node: character state. Dark circles: apomorphies. Open circles: homoplasies.

población de Caldera, en segundo lugar a las poblaciones intermareales distribuidas al norte de Antofagasta y por último, las poblaciones del desierto interior. La designación de tres especies se basa, por una parte, en la existencia de tres loci diagnóstico entre Caldera y las restantes poblaciones (EST-1, ESTD-1 y PGI-1), y por otro, por la ocurrencia de varias autapormorfías a nivel alélico para cada uno de los tres taxa específicos. Diferencias fijadas para dos loci es suficiente para afirmar que dos grupos corresponden a dos buenas especies (Baverstock \& Moritz 1996). Aunque no se pudo detectar alelos alternativos fijados entre las poblaciones de $M$. theresioides y M. quadrivittatus, existen alelos exclusivos para cada una de ellas, en algunos casos de alta frecuencia (alelo C de EST-1 y alelo D de AAT-2). La existencia de polimorfismos compartidos entre especies distintas, pero que poseen frecuencias alélicas marcadamente diferentes, es considerado un criterio válido por algunos autores para distinguir entre especies (Wiens 1993, Wiens \& Servedio 2000). Además, existen caracteres diagnósticos de tipo morfológico entre $M$. theresioides y $M$. quadrivittatus que indican que ambas son dos buenas especies (Ortiz 1980a, 1980b), a lo que se debe agregar sus marcadas diferencias ecológicas (alimentación y hábitat preferencial) (Ortiz \& Serey 1979, Heisig 1993). La asignación taxonómica de las poblaciones se basó fundamentalmente en sus distribuciones geográficas. Caldera es la localidad tipo de M. atacamensis, y Pica es la localidad tipo de M. theresioides. Aunque no se incluye la localidad tipo de la especie M. quadrivittatus, existen antecedentes que permiten afirmar que se trata de dicho taxón. Esta especie fue descrita por Tschudi en 1845. Debido al extravío temporal de la serie típica, DonosoBarros (1966) redescribió la especie asignándole un neotipo y restringiendo su localidad tipo a Iquique, localidad que sí se analiza en este trabajo. Sin embargo, posteriormente Ortiz (1989) redescubrió el material original recolectado por Tschudi en Islay en el sur de Perú, la cual debe revalidarse como localidad tipo para esta especie (Ortiz 1989). La escasa distancia geográfica entre Islay $\left(16^{\circ} 59^{\prime} \mathrm{S}, 72^{\circ} 06^{\prime} \mathrm{O}\right)$ y las localidades peruanas consideradas en este estudio (Ilo y Caleta Meca), junto a la semejanza morfológica que presentan estas poblaciones con las del norte de Chile, hacen plausible la idea de que son la misma especie. Las localidades del sur de Perú analizadas en este trabajo aparecen agrupadas fenética y filogenéticamente con las del extremo norte de Chile, y no existen diferencias genéticas como para afirmar que ambas son especies distintas.

Con respecto a los rangos de variabilidad genética, éstos pueden variar ampliamente en los 
herpetozoos en general, dependiendo del grupo que se trate. Los niveles de variabilidad genética observados en tropidurinos en particular comprenden rangos semejantes a los obtenidos para Microlophus. Martins (1995) encontró en los Tropidurus del grupo "nanuzae" del Brasil, niveles de polimorfismo entre un 11 y un $44 \%$, un promedio de alelos por locus entre 1,1 y 1,4 , y heterocigosis promedio desde $\mathrm{H}=0,002$ hasta un $\mathrm{H}=0,111$. Los valores obtenidos en este trabajo para los tres indicadores se encuentran dentro de los rangos para Tropidurus. La tendencia general dentro de las especies chileno-peruanas es a presentar valores altos de variabilidad en las poblaciones de interior, asignables a $M$. theresioides, pudiendo ser un atributo de esta especie que merece ser analizado en un contexto genético poblacional.

En el análisis cladista la mayor cantidad de diferencias apomórficas se observaron entre la población de Caldera (M. atacamensis) y las restantes poblaciones, incluidas las de interior (78\% de apoyo Jacknifing). Esto último permite proponer la existencia de dos grupos hermanos sustentados por cinco y cuatro autapomorfías respectivamente (Fig. 3, Anexo 2). De acuerdo a esto, nosotros encontramos evidencia de que los Microlophus de intermareal del grupo "peruvianus", distribuidos en el sur de Perú y norte de Chile, no conforman un grupo monofilético. Esto sugiere la existencia de un mayor grado de parentezco evolutivo entre las especies $M$. theresioides (de desierto interior) y M. quadrivittatus (del litoral norte), respecto a $M$. atacamensis. Esta última especie habría divergido tempranamente del clado anterior, lo que indica que es el taxón hermano del par (theresioidesquadrivittatus).

Existen dos probables explicaciones para dar cuenta de la existencia de hábitos intermareales en dos especies que pertenecen a distintos clados. Por una parte, la adaptación al hábitat costero pudo surgir independientemente en más de una oportunidad al interior del grupo peruvianus. En un momento evolutivo habría surgido en la especie M. quadrivittatus y en otro en M. atacamensis, correspondiendo así a un atributo homoplásico. Otra explicación es que esta característica pudo estar presente ya en el ancestro común del grupo, condición que se habría mantenido en dos de las tres especies derivadas, correspondiendo así a un atributo plesiomórfico.

No existen antecedentes acerca de los eventos de colonización histórica de los Microlophus en el norte de Chile ni de la antigüedad del grupo "peruvianus". Una aproximación al respecto es aportada por Heise (1998), quien concluyó a par- tir de análisis de ADN mitocondrial, que en las Islas Galápagos existen tanto especies del linaje "occipitalis" como de "peruvianus". Considerando los supuestos del reloj molecular, él estimó la edad de divergencia entre especies del continente y del archipiélago en aproximadamente 5 millones de años, de manera que el grupo peruvianus como tal existe al menos desde el Plioceno. Considerando el arreglo filogenético obtenido, es plausible la hipótesis de que un linaje ancestral habría avanzado desde Perú hacia el sur por la línea costera, usando ambientes intermareales más favorables que los áridos de interior. Una vez alli, este linaje se habría dividido en dos subgrupos: uno que dió origen a la especie $M$. atacamensis al sur de Antofagasta, y otro que pasó a ser el ancestro de las actuales $M$ quadrivittatus y $M$. theresioides. Un evento de colonización secundaria pudo ocurrir hacia el interior, probablemente a través de las quebradas transversales del norte de Chile, las que albergan ambientes de mayor humedad y disponibilidad de recursos. Éstas conectan el litoral con ambientes francamente desérticos de interior. Las poblaciones ancestrales de $M$. theresioides pudieron arribar desde la costa por estas rutas y posteriormente habrían divergido evolutivamente para originar una especie diferente. Esta explicación es más parsimoniosa que la alternativa de una colonización a través del desierto abierto, el cual ha sido un ambiente mucho más inhóspito desde hace más de 15 millones de años (Hinojosa \& Villagrán 1997). Actualmente existen poblaciones de Microlophus aisladas en quebradas, como es el caso de las detectadas en las quebradas Vittor y Azapa, las cuales son asignables a $M$. theresioides (J.C. Ortiz resultados no publicados).

El modo cómo habría divergido evolutivamente M. atacamensis del ancestro de las otras dos especies aquí analizadas, es difícil de explicar en el escenario geográfico actual. M. quadrivittatus y M. atacamensis se distribuyen a lo largo de un "hilo" litoral contínuo, y sin aparentes barreras que pudiesen impedir el flujo génico en el pasado. La explicación a esto requiere de cambios geomorfológicos ó climáticos que habrían generado una discontinuidad entre ambas especies. Actualmente existe una discontinuidad fitogeográfica en el desierto costero situada al sur de Antofagasta. Al norte de este límite se extiende el "desierto costero de Tocopilla" con características francamente áridas, y al sur se ubica el "desierto costero de Tal-Tal" más húmedo y que alberga zonas con mayor vegetación. Esta discontinuidad se prolonga hacia el interior para separar hacia el norte el «desierto interior» de naturaleza más árida, del "desierto estepario de las sierras 
costeras" hacia el sur (Gajardo 1994). Esta discontinuidad puede corresponderse con la existencia de condiciones ecológicas particulares, las que a su vez pudieron influir a través del tiempo en la especialización (y por ende disgregación) de los ancestros de las dos especies costeras. Otra explicación alude la probable ocurrencia de cambios en la forma de la línea de costa en el rango distribucional de ambas especies. La geología de la costa chilena sugiere que han ocurrido numerosos eventos de transgresión y regresión marina, asociadas a fenómenos de hundimientos y levantamientos (Cecioni 1970 ). Tales cambios pudieron generar irregularidades en el desarrollo de la línea de costa al sur de Antofagasta, aumentando la distancia entre poblaciones de Microlophus que actualmente aparecen como cercanas, de manera que en el pasado pudieron experimentar importantes reducciones del flujo génico entre ellas.

\section{AGRADECIMIENTOS}

Agradecemos a Jorge Moreno y Rodrigo Moreno por el aporte de ejemplares, a Viviane Jerez, Marcela Vidal y Martha Hengst por su colaboración en tareas de terreno. Aporte parcial del Proyecto de Instrumental Científico 2001, Dirección de Investigación, Universidad de Concepción. El presente trabajo fue financiado por el proyecto FONDECYT 1980115.

\section{LITERATURA CITADA}

BAVERSTOCK PR \& C MORITZ (1996) Project design. En: Hillis D, C Moritz \& BK Mable (eds) Molecular systematics, second edition: 17-27. Sinauer Associates, Sunderland, Massachusetts.

BUTH D (1984) The application of electrophoretic data in systematic studies. Annual Review of Ecology and Systematics 15: 501-522.

CECIONI G (1970) Esquema de paleogeografía chilena. Editorial Universitaria, Santiago, Chile. 144 pp.

DIXON J \& J WRIGHT (1975) A review of the lizards of the Iguanid genus Tropidurus in Perú. Contribution of Science, Natural History Museum, Los Angeles 271: $1-39$

DONOSO-BARROS R (1966) Reptiles de Chile. Editorial Universitaria, Santiago, Chile. 458 pp.

DUMÉRIL G \& A BIBRON (1837) Erpétologie générale ou histoire naturelle des complète reptiles librarie, tome $4^{\circ}$. Encyclopedique de Roret, Paris, France. 572 pp.

FARRIS J, V ALBERT, M KALLERSJO, D LIPSCOMB \& A KLUGE (1997) Parsimony jacknifing outperforms neighbour joining. Cladistics 12: 99-124.
FROST D (1992) Phylogenetic analysis and taxonomy of the Tropidurus group of lizards. (Iguania: Tropiduridae). American Museum Novitates 3033: 168.

GAJARDO R (1994) La vegetación natural de Chile. Editorial Universitaria, Santiago, Chile. $165 \mathrm{pp}$.

GOLOBOFF PA (1993) NONA. Version 2.0 (Windows). Computer program, distributed by the author.

HARRIS H \& DA HOPKINSONS (1976) Handbook of enzyme electrophoresis in human genetics. NorthHolland Publishing Company, Amsterdam, The Netherlands. 780 pp.

HEISE P J (1998) Phylogeny and biogeography of Galapagos lava lizards (Microlophus) inferred from nucleotide sequence variation in mitochondrial DNA. Ph.D. Dissertation, The University of Tennessee, Knoxville, Tennessee. 206 pp.

HEISIG M (1993) An ethological study of an island population of Tropidurus atacamensis. Salamandra 29: 65-81.

HINOJOSA L F \& C VILLAGRÁN (1997) Historia de los bosques del sur de Sudamérica, I: antecedentes paleobotánicos, geológicos y climáticos del Terciario del cono sur de América. Revista Chilena de Historia Natural 70: 225-239.

MARTINS JM (1995) Allozyme variation and expression in lizards of the Tropidurus nanuzae species group (Iguania: Tropiduridae). Copeia 1995: 665-675.

MERTENS R (1956) Zur Kenntnis der Iguaniden-Gattung Tropidurus in Peru. Senck Biologischen 37: 101-136.

MICKEVICH MF \& C MITTER (1981) Treating polimorphic characters in systematics: a phylogenetic treatment of electrophoretic data. Advances in Cladystics 1: 45-58.

MILLER MP (2000) Tools for population genetic analyses: a Windows program. Department of Biological Science, Northern Arizona University, Flagstaff, Arizona.

MURPHY R, JW SITES Jr, DG BUTH \& CH HAUFLER (1996) Proteins I: isozyme electrophoresis. En: Hillis DM, C Moritz \& BK Mable (eds) Molecular Systematics (Second edition): 51-129. Sinauer Associates, Sunderland, Massachusetts.

NEI M (1978) Estimation of average heterozygosity and genetic distance from a small number of individuals. Genetics 89: 583-590.

NIXON KC (2002) Winclada ver. 0.9.99.50.mexu (BETA). Published by the author, Ithaca, New York.

NORTHLAND I, J CAPETILLO, P ITURRA \& A VELOSO (1987) Estudios morfológicos y cromosómicos en el género Tropidurus (Iguanidae) del norte de Chile. Anales del Museo de Historia Natural de Valparaíso (Chile) 18: 115-122.

ORTIZ JC (1980a) Estudios comparativos de algunas poblaciones de Tropidurus de la costa chilena. Anales del Museo de Historia Natural de Valparaíso (Chile) 13: 267-280.

ORTIZ JC (1980b) Revisión taxonómica del género Tropidurus en Chile. Actas de la Primera Reunión Iberoamericana de Zoología de Vertebrados. Ediciones del Ministerio de Universidades e Investigación, La Rábida, España: 355-377. 
ORTIZ JC (1989) Catalogue des types du Musée d'histoire naturelle de nuchâtel. III. Sauriens. Bulletin de la Societé Neuchâteloise des Sciences Naturelles (France) 112: 47-64.

ORTIZ JC \& I SEREY (1979) Analisis factorial de correspondencia de las especies del género Tropidurus de Chile. Archivos de Biología y Medicina Experimentales (Chile) 12: 203-208.

RICHARDSON BJ, PR BAVERSTOCK \& M ADAMS (1986) Allozyme electrophoresis: a handbook for animal systematics and population studies. Academic Press, Sydney, Australia. 410 pp.

ROGERS JS (1972) Measures of genetic similarity and genetic distance. Studies in Genetics VII, University of Texas Publication 7213: 145-153.

SELANDER RK, MH SMITH, SY YANG, WE JOHNSON \& JR GENTRY (1971) Biochemical polymorphism and systematics in the genus Peromyscus I. Variation in the old-field mouse (Peromyscus polionotus). Studies in Genetics VI, University of Texas Publication 7103: 49-90.

SWOFFORD D \& G OLSEN (1990) Phylogeny reconstruction. En: Hillis DM \& C Moritz (eds) Molecular systematics: 441-501. Sinauer Associates, Sunderland, Massachussets.

TSCHUDI JJ (1845) Reptilium conspectus quae in Republic Peruana reperiuntur et pleraque observata vel collecta sunt in itenere. Archives Naturgeschland 2: 150-170.
VICTORIANO P, JC ORTIZ, L TRONCOSO \& R GALLEGUILLOS (1995) Allozyme variation in populations of Pleurodema thaul (Anura: Leptodactylidae). Comparative Biochemestry and Phisiology 112 B: 487-492.

WIENS J (1993) Phylogenetic systematics of the tree lizards (genus Urosaurus) Herpetologica 49: 399420

WIENS J (2000) Reconstructing phylogenies from allozyme data: comparing method performance with congruence. Biological Journal of the Linnean Society 70: 613-632.

WIENS J \& M SERVEDIO (2000) Species delimitation in systematics: inferring diagnostic differences between species. Proceedings of the Royal Society of London B 267: 631-636.

WILEY EO (1981) Phylogenetics. The theory and practice and phylogenetics systematics. John Wiley \& Sons, New York, New York. 439 pp.

YEH F, RC YANG, BJ THIMOTHY, Z-H YE \& J MAO (1997) POPGENE, the user-friendly shareware for population genetic analysis. Molecular Biology and Biotecnology Centre, University of Alberta, Alberta, Canada.

Editor Asociado: Milton Gallardo

Recibido el 16 de enero de 2001; aceptado el 29 de noviembre de 2002 


\section{ANEXO 1}

Descripción de los estados de caracteres aloenzimáticos utilizados en el análisis cladístico de los Microlophus (indicados en el Anexo 2). En los loci polimórficos los estados se basan en rangos de frecuencias de los alelos (entre paréntesis). No se incluyen los caracteres no informativos

Description of allozymic character states used in the phylogenetic analysis of Microlophus (showed in Appendix 2). In polymorphic loci states are based in frequency ranges of alleles (in parenthesis). Non informative characters are not shown

\begin{tabular}{|c|c|c|c|}
\hline Carácter & Estado & Carácter & Estado \\
\hline \multirow[t]{2}{*}{ 1: LDH-2 } & $0=$ alelo $\mathrm{B}$ fijado & \multirow[t]{4}{*}{ 19: MDHP-1 (A } & $0=0,00-0,25$ \\
\hline & $1=$ alelo A fijado & & $1=0,26-0,50$ \\
\hline \multirow[t]{4}{*}{ 2: EST-1 (A) } & $0=0,00-0,25$ & & $2=0,51-0,75$ \\
\hline & $1=0,26-0,50$ & & $3=0,76-1,00$ \\
\hline & $2=0,51-0,75$ & \multirow{2}{*}{ 20: MDHP-1 (B) } & $0=0,00-0,25$ \\
\hline & $3=0,76-1,00$ & & $1=0,26-0,50$ \\
\hline \multirow{4}{*}{ 3: EST-1 (B) } & $0=0,00-0,25$ & & $2=0,51-0,75$ \\
\hline & $1=0,26-0,50$ & \multirow{3}{*}{ 21: MDHP-1 (D } & $3=0,76-1,00$ \\
\hline & $2=0,51-0,75$ & & $0=0,00-0,25$ \\
\hline & $3=0,76-1,00$ & & $1=0,26-0,50$ \\
\hline \multirow[t]{4}{*}{ 4: EST-1 (C) } & $0=1,00-0,75$ & & $2=0,51-0,75$ \\
\hline & $1=0,74-0,50$ & \multirow{3}{*}{ 22: MDHP-1 (F) } & $3=0,76-1,00$ \\
\hline & $2=0,49-0,25$ & & $0=0,00-0,25$ \\
\hline & $3=0,24-0,00$ & & $1=0,26-0,50$ \\
\hline \multirow[t]{4}{*}{ 5: EST-1 (E) } & $0=0,00-0,25$ & \multirow{4}{*}{ 23: MDHP-1 (G } & $2=0,51-0,75$ \\
\hline & $1=0,26-0,50$ & & $3=0,76-1,00$ \\
\hline & $2=0,51-0,75$ & & $0=1,00-0,75$ \\
\hline & $3=0,76-1,00$ & & $1=0,74-0,50$ \\
\hline \multirow[t]{2}{*}{ 6: EST-2 } & $0=$ alelo B fijado & & $2=0,49-0,25$ \\
\hline & $1=$ alelo A fijado & & $3=0,24-0,00$ \\
\hline \multirow{4}{*}{ 7: AAT-1 (A) } & $0=0,00-0,25$ & \multirow[t]{4}{*}{ 24: PGM-1 (A) } & $0=0,00-0,25$ \\
\hline & $1=0,26-0,50$ & & $1=0,26-0,50$ \\
\hline & $2=0,51-0,75$ & & $2=0,51-0,75$ \\
\hline & $3=0,76-1,00$ & & $3=0,76-1,00$ \\
\hline 8: AAT-1 (C) & $0=1,00-0,75$ & 25: PGM-1 (C) & $0=1,00-0,75$ \\
\hline & $1=0,74-0,50$ & & $1=0,74-0,50$ \\
\hline & $2=0,49-0,25$ & & $2=0,49-0,25$ \\
\hline & $3=0,24-0,00$ & & $3=0,24-0,00$ \\
\hline 9: $\mathrm{AAT}-2(\mathrm{~A})$ & $0=0,00-0,25$ & 26: PGM-2 & $0=$ alelo $\mathrm{B}$ fijado \\
\hline & $1=0,26-0,50$ & & $1=$ alelo $\mathrm{A}$ fijado \\
\hline & $2=0,51-0,75$ & 27: LAP-1 & $0=$ alelo $\mathrm{B}$ fijado \\
\hline & $3=0,76-1,00$ & & $1=$ alelo $\mathrm{A}$ fijado \\
\hline 10: AAT-2 (B) & $0=0,00-0,25$ & 28: ESTD-1 (A) & $0=0,00-0,25$ \\
\hline & $1=0,26-0,50$ & & $1=0,26-0,50$ \\
\hline & $2=0,51-0,75$ & & $2=0,51-0,75$ \\
\hline & $3=0,76-1,00$ & & $3=0,76-1,00$ \\
\hline 11: AAT-2 (C) & $0=1,00-0,75$ & 29: ESTD-1 (B) & $0=0,00-0,25$ \\
\hline & $1=0,74-0,50$ & & $1=0,26-0,50$ \\
\hline & $2=0,49-0,25$ & & $2=0,51-0,75$ \\
\hline & $3=0,24-0,00$ & & $3=0,76-1,00$ \\
\hline 12: AAT-2 (D) & $0=0,00-0,25$ & 30: ESTD-1 (C) & $0=0,00-0,25$ \\
\hline & $1=0,26-0,50$ & & $1=0,26-0,50$ \\
\hline & $2=0,51-0,75$ & & $2=0,51-0,75$ \\
\hline & $3=0,76-1,00$ & & $3=0,76-1,00$ \\
\hline 13: AAT-2 (F) & $0=1,00-0,75$ & 31: ESTD-1 (D) & $0=1,00-0,75$ \\
\hline & $1=0,74-0,50$ & & $1=0,74-0,50$ \\
\hline & $2=0,49-0,25$ & & $2=0,49-0,25$ \\
\hline & $3=0,24-0,00$ & & $3=0,24-0,00$ \\
\hline 14: IDH-1 (A) & $0=0,00-0,25$ & 32: ESTD-1 (E) & $0=0,00-0,25$ \\
\hline & $1=0,26-0,50$ & & $1=0,26-0,50$ \\
\hline & $2=0,51-0,75$ & & $2=0,51-0,75$ \\
\hline & $3=0,76-1,00$ & & $3=0,76-1,00$ \\
\hline 15: IDH-1 (D) & $0=0,00-0,25$ & 33: ESTD-2 & $0=$ alelo $\mathrm{C}$ fijado \\
\hline & $1=0,26-0,50$ & & $1=$ alelo $B$ fijado \\
\hline & $2=0,51-0,75$ & & $2=$ alelo $\mathrm{A}$ fijado \\
\hline & $3=0,76-1,00$ & 34: PGI-1 (A) & $0=1,00-0,75$ \\
\hline 16: IDH-1 (E) & $0=1,00-0,75$ & & $1=0,74-0,50$ \\
\hline & $1=0,74-0,50$ & & $2=0,49-0,25$ \\
\hline & $2=0,49-0,25$ & & $3=0,24-0,00$ \\
\hline & $3=0,24-0,00$ & 35: PGI-1 (B) & $0=0,00-0,25$ \\
\hline 17: IDH-2 & $0=$ alelo A fijado & & $1=0,26-0,50$ \\
\hline & $1=$ alelo B fijado & & $2=0,51-0,75$ \\
\hline 18: GPD-1 & $0=$ alelo $\mathrm{B}$ fijado & & $3=0,76-1,00$ \\
\hline & $1=$ alelo A fijado & 36: PGI-1 (C) & $0=0,00-0,25$ \\
\hline & & & $1=0,26-0,50$ \\
\hline & & & $2=0,51-0,75$ \\
\hline & & & $3=0.76-1.00$ \\
\hline
\end{tabular}




\section{ANEXO 2}

Matriz de estados de los 36 caracteres polarizados en los que se basó el análisis filogenético de las poblaciones de Microlophus. Basada en el Anexo 1. (0) carácter plesiomórfico; (1), (2) y (3) estados apomórficos o derivados

Data matrix of states of 36 character used in phylogenetics analyses of populations of Microlophus. Based in Appendix 1. (0) plesiomorphic character; (1), (2) and (3) apomorphic state

\begin{tabular}{|c|c|c|c|c|c|c|c|}
\hline & 1 al 5 & 6 al 10 & 11 al 15 & $\begin{array}{l}\text { Carácter } \\
16 \text { al } 20\end{array}$ & 21 al 25 & 26 al 30 & 31 al 36 \\
\hline occipitalis & 00000 & 00000 & 00000 & 00000 & 00000 & 00000 & 000000 \\
\hline thoracicus & 10033 & 13330 & 00303 & 31100 & 30333 & 11000 & 331303 \\
\hline Ilo & 13030 & 11230 & 00330 & 30120 & 01333 & 11030 & 302000 \\
\hline Caleta Meca & 13030 & 10030 & 00330 & 30130 & 00333 & 11030 & 302000 \\
\hline Arica & 13030 & 13330 & 00330 & 30130 & 00333 & 11030 & 302000 \\
\hline Iquique & 13030 & 13330 & 00330 & 30130 & 00333 & 11030 & 302000 \\
\hline Loa & 13030 & 13330 & 00330 & 30130 & 00333 & 11030 & 302110 \\
\hline Antofagasta 1 & 13030 & 13320 & 00330 & 30101 & 00333 & 11030 & 302000 \\
\hline Antofagasta 2 & 13030 & 13300 & 20330 & 30121 & 00333 & 11030 & 302000 \\
\hline Caldera & 10330 & 13303 & 00330 & 30103 & 00333 & 11300 & 302330 \\
\hline Pica & 12020 & 13330 & 00330 & 30102 & 00333 & 11021 & 302000 \\
\hline Huayca & 11020 & 13310 & 02330 & 30120 & 00333 & 11030 & 302000 \\
\hline Mamiña & 11020 & 13320 & 01330 & 30111 & 00333 & 11030 & 302000 \\
\hline
\end{tabular}

